\title{
The Situation of HIV/M. tuberculosis Co-Infection in India
}

\author{
Pradeep Seth ${ }^{*}$
}

Seth Research Foundation, 1, Aradhana Colony, Sector 13, R.K. Puram, Ring Road, New Delhi-110066, India

\begin{abstract}
In November 2008, European Commission initiated a collaborative research and information dissemination project entitled "European Network for global cooperation in the field of AIDS and TB (EUCO-Net)" involving Institutions in Europe (Germany, Belgium, Italy), Latin America (Brazil, Argentina, Colombia), Russia, South Africa, and India with the following objectives: a) to provide an overview of the state of art in HIV and TB research and disease management in different partner countries; b) to identify global research priorities; and c) to boost International cooperation between leading HIV and TB experts from Europe and those countries mainly affected by these two diseases. Therefore, in this report from India these objectives have been addressed under the following topics: i) Basic demographic data; ii) Basic epidemiological Data of HIV and TB; iii) Medical treatment standards; iv) Diagnostic Standards.
\end{abstract}

Keywords: India, HIV/M. tuberculosis co-infection, revised national TB control programme (RNTCP).

\section{INTRODUCTION}

The first HIV estimation in India was done in 1994 based on data from 52 sites. Since then, the process of estimation of HIV infected persons in the country has evolved to a very great extent. Since, the samples from which data were collected through sentinel surveillance were not exactly representative of the general population certain assumptions were used to generate estimates for the general population. Over the years, these assumptions were gradually refined with the help of other available data sources. The year 2006 provided a unique opportunity when multiple data sources such as a community based HIV prevalence study of National Family Health Survey-III, Integrated Biobehavioural Assessment Survey, Endline Behavioural Surveillance Survey could be utilized along with the data from the expanded sentinel surveillance system to arrive at more robust HIV estimates that are closer to reality. Moreover, in 2006, the Workbook Model of WHO-UNAIDS was adopted that allowed international comparability. Special statistical packages such as Random effects model and Spectrum Projection Software are utilized to make more accurate and reliable estimates.

The burden of MTB/TB and HIV/AIDS pose unprecedented challenges on the public health system in India. Globally, India has the highest burden of TB and the number of HIV-infected people estimated in India is second highest after South Africa. In addition to morbidity and mortality, the two diseases cause substantial economic and social burden on the nation. TB and HIV are overlapping epidemics. The HIV pandemic presents a massive challenge to the control of TB at all levels. People living with HIV have increased susceptibility to active tuberculosis, and HIV infection is the greatest risk factor worldwide for tuberculosis disease. TB is also one of the most common

\footnotetext{
*Address correspondence to this author at the Seth Research Foundation, 1, Aradhana Colony, Sector 13, R.K. Puram, Ring Road, New Delhi-110066, India; Tel: +91-9810311407; Fax: +91-11-26876639;
}

E-mail: seth.res.fdn@gmail.com causes of morbidity and one of the leading causes of mortality in people living with HIV/AIDS.

In recent years, India has witnessed rapid expansion of the DOTS strategy for TB control and scaling up of interventions to combat HIV/AIDS, including access for treatment with antiretroviral drugs. With the establishment of new mechanisms of funding like the Global Funds for AIDS, TB and Malaria, financing of interventions against MTB/HIV is no longer an issue. Additionally, over the last few years, considerable experience has been gained on TB and HIV programme coordination, as well as individual patient care. Progress in improving HIV/MTB coordination and linkages in service delivery will contribute greatly to achieving these goals.

\section{BASIC DEMOGRAPHIC DATA}

The demographical information of India was obtained from a website "Census of India" [1]. The information collected during a countrywide census conducted in 2001. Census in India is undertaken every ten years.

India is a large country comprising 28 states and 7 union territories. These states and the union territories are divided into districts. At the time of Census 2001 there were in all 593 districts. Each district is further divided in to sub-districts, which are known differently in different parts in the country.

As of 1st March, 2001 the population of India stood at 1,027,015,247 comprising 531,277,078 males and 495,738, 169 females. Thus, India becomes the second country in the world after China to cross the one billion mark. Life expectancy at birth is 64 years. Urban population constitutes only $27.2 \%$ of the total population. Meaning thereby, almost three-fourth's of India's population lives in rural areas. One-third of India's households are in urban areas, with two-thirds in rural areas.

\section{BASIC EPIDEMIOLOGIC DATA}

\section{Method}

In India, countrywide control and prevention of HIV/AIDS and Tuberculosis is the responsibility of the Government of India, Ministry of Health and Family 
Welfare (MOHFW). Two independent departments have been created under the MOHFW to achieve the desired effect. National AIDS Control Organization (NACO) is responsible for control and prevention of HIV/AIDS; and Central TB Division of the Directorate General of Health Services, looks after tuberculosis prevention and control. Epidemiological data released by these sources were chosen because they were based on sound epidemiological methods accepted by the WHO and UNAIDS. The data were comprehensive and permitted comparison with global data released by the WHO and UNAIDS.

Epidemiological data on HIV/AIDS were obtained from NACO report on HIV sentinel surveillance and HIV estimation in India-2007 [2] and Annual Report NACO 2008-09 [3]. The data on Tuberculosis (TB) and multiple drug resistance tuberculosis MDR) were obtained from the reports from Central TB Division $[4,5]$.

HIV/AIDS epidemiologic data from NACO did not stratify the estimated prevalence data as per EUCO-Net questionnaire requirement particularly prevalence of HIV infection in children ( 0 to 12 years). The cut-off age as per NACO document is $<15$ years for children. Therefore in the present report also we used $<15$ years cut-off age for disaggregation of data between children and adults.

Epidemiological data on TB were obtained from Revised National Tuberculosis Control Programme (RNTCP) Report -2009 [4]. Here also, Central TB Division did not stratify the estimated prevalence data age-wise. Indeed, it is only from the year 2006 that the pediatric patients are being covered under RNTCP. In addition, other reports of the Central TB Division like Technical and operational Guidelines of TB control [6] Pediatric Guidelines, RNTCP Guidelines for diagnosis of TB [7], RNTCP DOTS plus guidelines [8] etc. were consulted.

\section{Epidemiology}

\section{HIV-AIDS Epidemiology}

The data generated through HIV sentinel surveillance is used to estimate the level of infections in the country at regular intervals. The annual surveillance and estimation helps to understand the course of epidemic stage in different

\section{HIY Prevalence \%}

regions. NACO utilizes this information for effective planning and implementation of its programs. Standardized methods supported by WHO/UNAIDS are employed for estimating the burden of the epidemic overtime. Adult HIV prevalence has been estimated using WHO/UNAIDS Workbook restructured in 2006 for Indian epidemic situation. It included five subpopulations: intravenous drug users (IDU), men having sex with men (MSM), female sex workers (FSW), long distance truckers and the general population represented by the antenatal clinic attendees. Age-wise stratification of data was done by Spectrum software.

The total number of people living with HIV/AIDS (PLHA) in India in 2007 is estimated to be 2.31 million (1.82.9 million, Fig. 1). Females constitute around $39 \%$ of the burden (0.9 million). Children below 15 years constitute $3.5 \%$ of the estimated number of PLHA while elderly people with age greater than 49 years constitute $7.8 \%$. Adults aged $15-49$ years constitute $88.7 \%$ of the estimated number of PLHA. The highest number of PLHA is in Andhra Pradesh and Maharashtra, with nearly half-a-million PLHA each. Besides Tamil Nadu and Karnataka, West Bengal, Gujarat and Uttar Pradesh are estimated to have higher burden of the epidemic with greater than 0.1 million PLHA in each of these states. The four South Indian states contribute $60 \%$ of all PLHA in the country and along with West Bengal, Gujarat and Uttar Pradesh they contribute $80 \%$ of PLHA in India. Though Manipur and Nagaland have the highest HIV prevalence in the country, due to small population size, the estimated number of PLHA in these two states is less than 25,000. The states of Kerala, Bihar and Rajasthan have more than 50,000 PLHA each though the HIV prevalence in these states is low. Fig. (2) shows the distribution of PLHA among the high burden states of India.

Estimated Adult HIV prevalence in India in 2007 is $0.34 \%(0.25 \%-0.43 \%)$ (Fig. 1). Estimated HIV prevalence among males $(0.40 \%)$ continues to be higher than among females $(0.27 \%)$. Estimated Adult HIV prevalence remains $>1 \%$ in Manipur (1.57\%) and Nagaland (1.20\%) in 2007. Andhra Pradesh has an estimated adult HIV prevalence of $0.97 \%$ while Karnataka and Maharashtra have estimated adult HIV prevalence $<1 \%$. Tamil Nadu, West Bengal,

Total PLHA
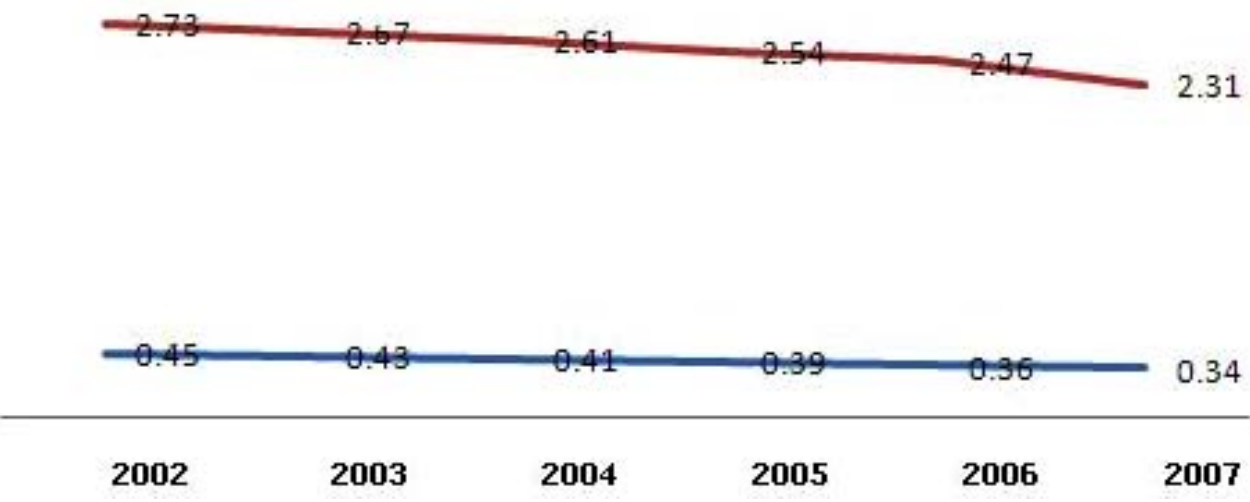

Fig. (1). Estimated Adult HIV Prevalence and total number of PLHA (million) in India 2002-07 (Adapted from HIV Sentinel Surveillance Report). 


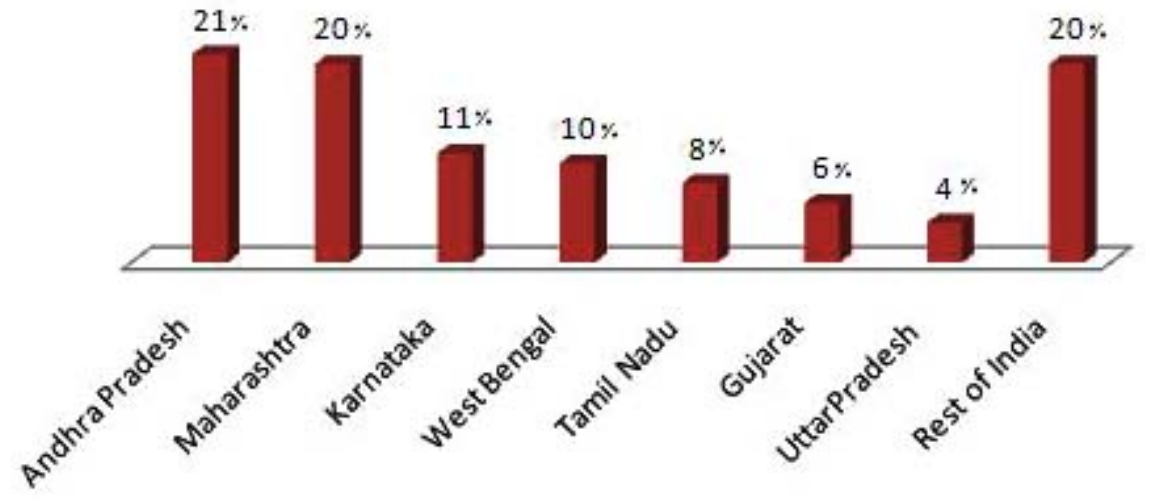

Fig. (2). Distribution of PLHA among High Burden States (2007) (Adapted from Annual Report NACO).

Gujarat and Delhi have estimated adult HIV prevalence of $0.4 \%$. Fig. (2) shows the year-wise estimated adult HIV prevalence from 2002 to 2007 derived from Spectrum Package. Fig. (3) shows the state-wise estimated adult HIV prevalence in selected groups for the years 2006 and 2007 derived from Workbook model as well as Spectrum Package.

However, there are considerable differences in the prevalence rates across different geographical regions. HIV Prevalence amongst ANC clinic attendees has been around $1 \%$ in the states of Andhra Pradesh, Karnataka in South India and in Manipur, Nagaland and Mizoram in Northeastern region of India. Tamil Nadu and Maharashtra have recorded less than $1 \%$ HIV prevalence in ANC clinic attendees. The epidemic is greater in urban areas than rural areas, greater among males than females, decreases with increasing education level, and is found to be highest among women whose spouses work in transport industry.

\section{HIV Epidemic Among High Risk Groups}

\section{Female Sex Workers}

At the state level, HIV prevalence among FSWs is very high in Maharashtra (17.91\%), followed by Manipur (13.07\%), Andhra Pradesh (9.74\%), Nagaland (8.91\%) and Mizoram (7.2\%). Among the other states, Gujarat, Karnataka, and West Bengal have HIV prevalence greater than 5\% among FSW. Fig. (3) shows state-wise HIV prevalence among high risk groups. Pockets in three cities of
Maharashtra state: Pune, Mumbai and Thane have shown $>30 \%$ HIV prevalence among FSW.

However, there is a decline in HIV prevalence among FSW in South Indian States reflecting the impact of interventions, while rising trends are evident in the North East suggesting a dual nature of the epidemic. In the low prevalence states, the trends are stable.

\section{Men who have Sex with Men}

Expansion of surveillance among MSM has revealed new pockets of epidemic. Among MSM, high HIV prevalence is recorded in the states of Karnataka $(17.6 \%)$ followed by Andhra Pradesh (17.04\%), Manipur (16.4\%), Maharashtra $(11.80 \%)$ and Delhi $(11.73 \%)$, Goa $(7.93 \%)$ and Gujarat $(8.40 \%)$ (Fig. 3). In total, 10 states have shown greater than $5 \%$ HIV prevalence among MSM. Thirty out of forty districts with MSM sites show HIV prevalence greater than $5 \%$. Among MSM, HIV trends are rising in south Indian states. Rising trends are also noted Delhi while trends are stable at the single MSM site in Manipur.

\section{STD Clinic Attendees}

Among the STD clinic attendees, Andhra Pradesh continues to show the highest prevalence (19.72\% $(7.60 \%$ $39.20 \%$ ) followed by Maharashtra (16.18\% (7.20\%-32.20\%), Karnataka $(7.15 \% \quad(1.60 \%-10.80 \%))$ and Tamil Nadu $(12.04 \%$ (1.60\%-38.40\%)). Mizoram (7.13\%) and Goa

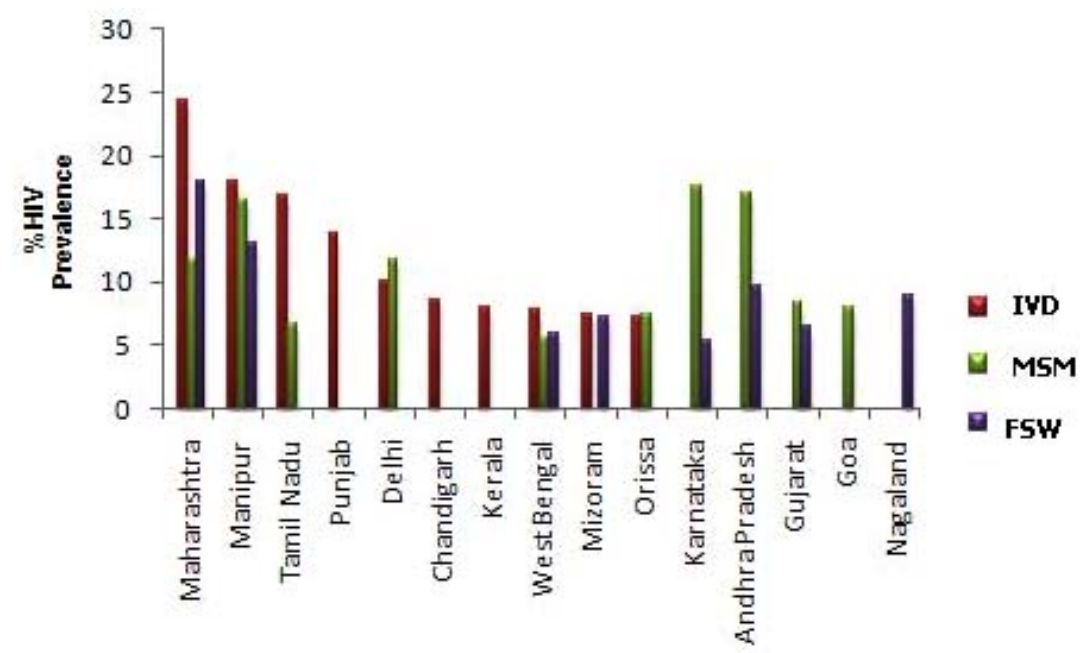

Fig. (3). States with high prevalence among different groups, 2007; IVD, injecting drug users; MSM, men who have sex with men; FSW, female sex workers (Adapted from HIV Sentinel Surveillance Report). 
$(5.60 \%)$ have also shown HIV prevalence greater than 5\% among STD clinic attendees. Trends among STD clinic attendees are declining at all India level and in high prevalence states, while rising trends are noted in Chhattisgarh, Mizoram and Gujarat. Stable trends are noted in other low prevalence states.

\section{Injecting Drug Users}

High HIV prevalence among IDUs has been noted in Maharashtra (24.4\%), Manipur (17.90\%), Tamil Nadu $(16.80 \%)$, Punjab (13.79\%), Delhi (10.10\%), Orissa (7.3\%) and Kerala \& West Bengal (7.8\%). Trends among IDUs are on a decline in Manipur, Nagaland and Chennai reflecting impact of interventions while rising trends are noted in Meghalaya, Mizoram, West Bengal, Mumbai, Kerala and Delhi.

The overall HIV prevalence among different population groups in 2007 continues to portray the concentrated epidemic in India, with a very high prevalence among High Risk Groups - IDU (7.2\%), MSM (7.4\%), FSW (5.1\%) \& STD clinic attendees (3.6\%) (Fig. 4) and low prevalence among ANC clinic attendees (age adjusted - $0.48 \%$ ).

Though heterosexual mode of transmission is still the predominant mode of HIV transmission in India, HIV epidemic in India appears to be dual epidemic driven by sexual and IDU routes of transmission, concentrated in nature with high HIV prevalence among high risk groups and heterogeneous in spread with pockets of infection found in various districts of the country.

\section{Tuberculosis Epidemiology}

India is the highest TB burden country globally (29.9 million patients), accounting for one fifth of the global incidence and 2/3rd of the cases in South East Asia (Fig. 5). Nearly $40 \%$ of the Indian population is infected with the MTB bacillus. Each year, 1.9 million new cases of TB occur in the country, of which about 0.8 million are infectious new smear positive pulmonary TB cases. The estimate of TB incidence in India is based on findings of the nationwide Annual Risk of MTB Infection (ARTI) study conducted in 2000-03. The national ARTI was estimated at $1.5 \%$ i.e. 75 new smear positive pulmonary TB cases are expected per 100,000 population annually. The prevalence of TB has been estimated at 3.8 million bacillary cases for the year 2000, by the expert group of Govt. of India. In addition, an estimated $40 \%$ of the Indian population is latently infected with $M$. tuberculosis.

By any measure the burden of TB in India is staggering. More than $80 \%$ of the burden of tuberculosis is due to premature death, as measured in terms of disability-adjusted life years (DALYs) lost. Every day, more than 5,000 people develop TB disease, and nearly 1,000 people die of TB, i.e. 2 deaths every 3 minutes. As per WHO estimates in 2006, nearly 322,000 persons in India died of tuberculosis (mortality rate 28 per 100,000 persons), which was estimated at over 500,000 annually at the beginning of the RNTCP. Data from specific surveys, however, suggest that case fatality rates prior to RNTCP were generally greater than $25 \%$. In the RNTCP era, case fatality has remained below $5 \%$ for new cases registered for treatment under the programme. Deaths due to TB exceed the combined deaths from all other communicable diseases and account for $26 \%$ of all avoidable adult deaths. TB is also the leading killer of women, causing more orphans than those produced by all causes of maternal mortality combined.

\section{MDR/XDR Tuberculosis}

The emergence of resistance to drugs used to treat TB, and particularly MDR-TB, has become a significant public health problem in a number of countries and an obstacle to effective TB control. Several small surveys conducted across the country have shown the prevalence rates of MDR-TB in the country at around 1\%-3\% among new cases, and $12 \%$ among retreatment cases. A retrospective analysis of various randomized clinical trials conducted by the Tuberculosis Research Centre (Chennai, India) with various rifampicin containing regimens in the initial intensive phase, and with or without rifampicin in the continuation phase, revealed an overall emergence of resistance to rifampicin in only $2 \%$ patients despite a high level $(18 \%)$ of initial resistance to isoniazid either alone or in combination with other anti-TB drugs. A large scale population based survey in the states of Gujarat and Maharashtra has also indicated similar resistance levels (new-3\% and retreatment-12-17\%). Available information suggests that the proportion of MDR-TB is relatively low in India. However, this translates into a large absolute number of cases, with an estimated annual

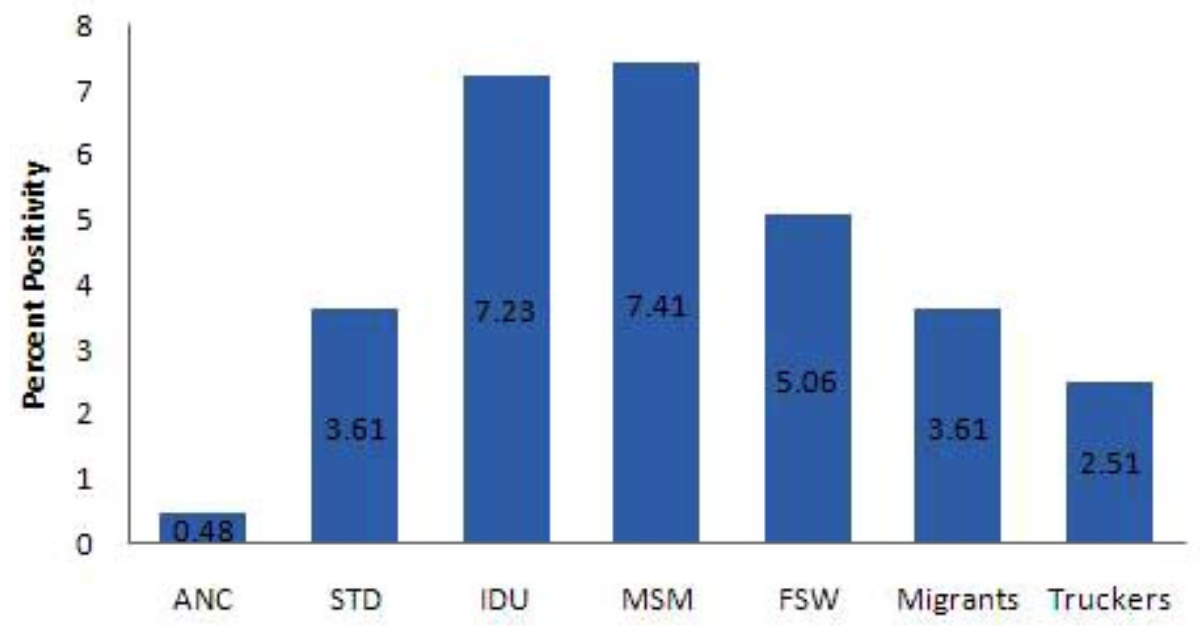

Fig. (4). HIV prevalence among different population groups (Adapted from Annual Report NACO). 

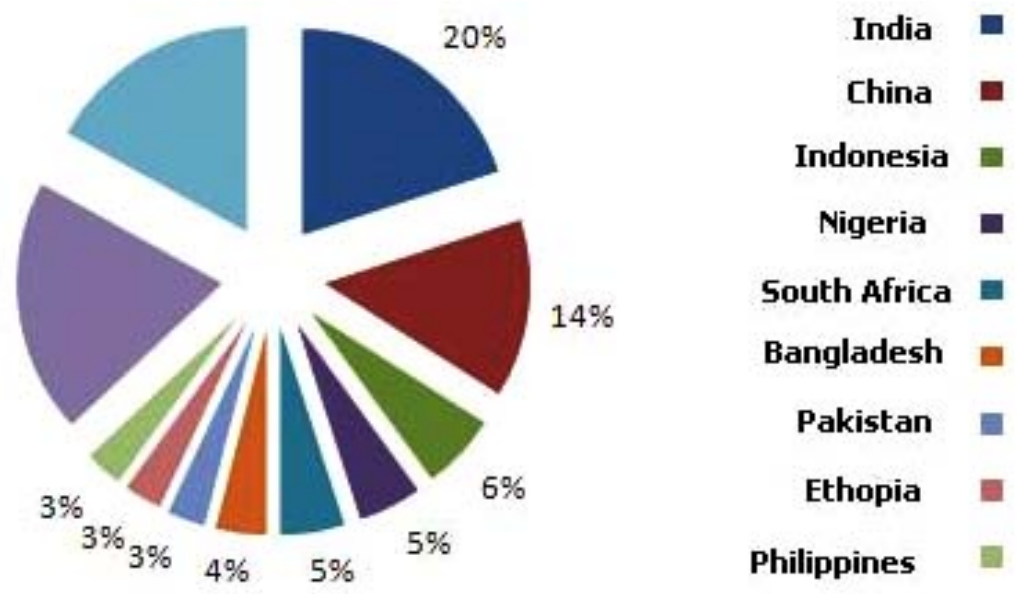

Fig. (5). Global Incidence of TB Burden (Adapted from RNTCP Status Report).

incidence of 110,000 cases of MDR-TB. XDR-TB has been reported in India by isolated studies with non-representative and highly selected clinical samples. The magnitude of the problem remains to be determined due to the absence of laboratories capable of conducting quality assured second line Drug Susceptibility Testing (DST). However, what is frightening is the potential threat of XDR-TB in India, with unregulated availability and injudicious use of the second line drugs along with non-existence of systems to ensure standardized regimens and treatment adherence for MDR-TB outside the national programme. The problem of MDR and XDR-TB in India and across the world raises the possibility that the current TB epidemic of mostly drug susceptible TB will be replaced with a form of TB with severely restricted treatment options. If this happens it would jeopardize the progress made in recent years to control TB globally as well as in India and would also put at risk the plans to progress towards a world where TB ceases to be a public health problem.

A major limiting factor in making State wise representative data on MDR/XDR-TB is the lack of quality assured culture and drug sensitivity testing (DST) laboratory facilities. It is important to note that the diagnosis of MDR/XDR-TB is laboratory based. RNTCP is in the process of establishing in a phased manner a nationwide network of quality assured culture and DST laboratory facilities.

\section{BCG Vaccination}

In India BCG vaccination is a part of Universal Immunization Programme (UIP) under which vaccines for six preventable diseases (tuberculosis, diphtheria. Pertusis, tetanus, poliomyelitis and measles) are available to all eligible children free of cost. This programme was launched in 1985 with the aim to cover all districts by 1990 and to immunize $85 \%$ of all infants against six vaccine preventable diseases. BCG vaccination has a complementary role in TB control, with particular impact in the prevention of severe forms of TB in children [9]. The vaccination has a nonspecific beneficial effect on infant survival and a BCG scar is a marker of better survival among children in areas with high child mortality. Important contributing factors for the variable efficacy observed for the present BCG vaccine are said to include background immunity induced by nontuberculous environmental mycobacteria, diversity of BCG strains, and over- attenuation of presently used strains.
However, BCG vaccination has no effect on the transmission of the disease. The most effective step in the prevention of TB is to cure infectious cases in order to break the chain of transmission. The BCG Vaccination is given at birth under UIP to all children including HIV infected child if the child is asymptomatic or mildly symptomatic. The guidelines issued by the NACO, recommend withholding all live vaccines for symptomatic and severely immunecompromised HIV infected children.

\section{MEDICAL TREATMENT STANDARDS}

\section{HIV Medical Treatment Standards}

The free ART programme was launched on 1 April 2004 by the Government of India. The technical guidelines were initially modified from "WHO guidelines for ART in resource constraint settings". As the global experience increased a need was felt to revise the 2004 guidelines. The final guidelines were developed which were again modified in view of revised WHO 2006 ART guidelines [10]. As recommended in these guidelines any person who has a confirmed HIV infection is subjected to further evaluation for determining whether he requires ART or not by performing CD4 count and other baseline investigations. All those eligible as per technical guidelines are started on ART. Initially eight tertiary-level government hospitals in the six high-prevalence states of Andhra Pradesh, Karnataka, Maharashtra, Tamil Nadu, Manipur and Nagaland, as well as the NCT of Delhi were included in this programme. In Phase I of the implementation of this programme, the subgroups of the people living with HIV/AIDS (PLHA) targeted on a priority basis were: i) sero-positive mothers who have participated in the prevention of parent-to-child transmission (PPTCT) programme; ii) sero-positive children below the age of 15 years; and iii) people with AIDS who seek treatment in government hospitals. The ART centres were scaled up in a phased manner and to provide treatment to 100,000 patients by the end of 2007 and 300,000 patients by 2011 in 250 centres across the country.

The free ART programme has adopted the public health approach to administration and distribution of ART all of which are manufactured in India. This implies a comprehensive prevention, care and treatment programme, with a standardized, simplified combination of ART regimens, a regular secure supply of good-quality ARV 
drugs, and a robust monitoring and evaluation system. Since all first line and second line ART drugs are being manufactured in India the Government of India has been able to scale up ART under NACO with the aim to provide care and treatment to as many people as possible, while working towards universal access to care and treatment. The selection of first-line regimens is determined on the basis of a number of considerations, such as potency, profile of side-effects, ability to keep future treatment options open, ease of adherence, cost, risk during pregnancy and potential of the development of resistant viral strains. The current global recommendation in all circumstances is a triple drug regimen.

The national ART programme aims at i) providing longterm ART to eligible patients, ii) monitoring and reporting treatment outcomes on a quarterly basis; iii) attaining individual drug adherence rates of $95 \%$ or more; iv) increaseing life span so that $50 \%$ of patients on ART are alive 3 years after starting the treatment; and v) ensuring that $50 \%$ of patients on ART are engaged in or can return to their previous employment.

\section{Eligibility for $A R T$}

The national programme offers ART to the following groups of persons: i) All persons with HIV infection who are clinically eligible to receive ART; and ii) those who are already on ART (outside the national programme) and want to enrol with the national programme for the available ART regimens, after written informed consent.

\section{Antiretroviral Therapy Regimens}

Currently, the national programme provides the following combinations for first-line regimens

$$
\text { Stavudine (30 mg) + Lamivudine (150 mg) }
$$

$$
\text { Zidovudine (300 mg) + Lamivudine (150 mg) }
$$

(iii) Stavudine $(30 \mathrm{mg})+$ Lamivudine $(150 \mathrm{mg})+$ Nevirapine $(200 \mathrm{mg})$

(iv) Zidovudine $(300 \mathrm{mg})+$ Lamivudine $(150 \mathrm{mg})+$ Nevirapine $(200 \mathrm{mg})$

(v) Efavirenz (600 mg)

(vi) Nevirapine (200 mg)

Current NACO treatment guidelines for first-line ART recommends two classes of drugs for initial treatment ie 2 NRTI + 1 NNRTI. Fixed-dose combinations (FDCs) are preferred because they are easy to use, have distribution advantages (procurement and stock management), improve adherence to treatment and thus reduce the chances of development of drug resistance. The current national experience shows that bid (twice a day) regimens of FDCs are well tolerated and complied with.

Initiative to provide free ART to pediatric patients was launched on Nov 30, 2006 by NACO. So far 47,784 children living with HIV/AIDS (CLHA) have registered at ART centres and 14,383 CLHA have been receiving free ART as on March 2009.

Since monitoring of patients on first line ART is done by periodic peripheral blood CD4 T cell count at ART centres, data on emerging ART resistant strains are not available with
NACO. Nonetheless, NACO has developed guidelines in 2008 on roll out of second line ART [11]. According to these guidelines failure of first line ART should be suspected among the patients who having received first line ART for at least six months either show clinical deterioration after initial improvement or no improvement despite good adherence to therapy. The guidelines further define ART failure as clinical, immunological (fall in CD4 count to pre-therapy level or persistent CD4 levels below 100 cells/ul), and virological (plasma viral load $>10,000$ copies $/ \mathrm{mL}$ ).

After the failure of first line therapy the second-line ART is the next regimen immediately used in sequence. Second line ART was initiated in January 2008 at only two site (Tamil Nadu and Mumbai). During following 12 months it was expanded to 10 more centres. Though provision was made to provide second line drugs to 3000 patients during 2008-09, only 344 patients were receiving second line drugs. The NACO standard second line regimen comprise Tenofovir DF + Lamivudine+ Zidovudine+ Lopinavir/Ritonavir (TDF + 3TC + ZDV + LPV/r) aims to achieve viral suppression for as long as possible, so that survival can be prolonged. Current NACO treatment guidelines recommend that the protease inhibitor (PI) class is reserved for, and therefore characterizes second-line ART.

\section{Transmission of Drug Resistant HIV Strains}

There is no study from NACO on this subject. Studies from academic institutions on small sample size suggest about $10 \%$ primary infections may be due to resistant strains (Seth P. unpublished). Nevertheless, there is low prevalence of primary drug resistant infections. This scenario offers an opportunity to maintain low levels of drug resistant HIV strains by provision of better treatment monitoring. However, compliance is an issue in patients undergoing ARV treatment even though it is free. Therefore, there is likelihood of rise in drug resistant HIV strains and their subsequent transmission.

\section{Tuberculosis Medical Treatment Records}

RNTCP launched in 1997 is based on DOTS strategy. RNTCP uses intermittent short-course chemotherapy (SCC) regimens to facilitate direct observation of treatment. This is consistent with the World Health Organization guidelines. RNTCP ensures that there is no interruption in drugs and treatment once a person is diagnosed with TB. Sufficient anti-TB drugs in patient-wise boxes are made available at all the appropriate levels (Peripheral Health Institution/TB unit/District/ State/National) ensuring that the treatment does not stop mid-way due to lack of drugs.

The uninterrupted supply of drugs to each patient is made possible through the "patient-wise box." Patient-wise drug boxes (both adult and pediatric) are an innovation of RNTCP wherein a box of medications for the entire duration of the treatment is earmarked for every patient registered. This ensures the availability of the full course of medication to the patient the moment s/he is registered for treatment. Patientwise drug boxes have helped to improve patient care, adherence, drug supply and drug stock management.

Under RNTCP, all sub-centres, primary health centres, community health centres, and other health facilities provide DOTS services to patients. Since TB patients may also seek 
treatment from private physicians, the government has taken initiatives to provide DOTS services through the private sector and through community volunteers.

By March 2006, 1114 million populations have been covered in a nationwide programme for tuberculosis detection. So far 10 million patients have been treated with a success rate of $86 \%$, reducing the death rate due to tuberculosis from $29 \%$ in 1990 to $4 \%$ in 2006 .

\section{Direct Observed Treatment (DOTS and DOTS-Plus)}

Directly observed treatment (DOT) is one of the key elements of the DOTS strategy. Under DOTS programme RNTCP uses four oral anti-TB drugs (Isoniazid, Rifampicin, Ethambutol and Pyrazinamide). In DOTS, an observer (health worker or trained community volunteer who is not a family member) watches and supports the patient in taking drugs. It is this DOTS provider who ensures that the patient takes the right drugs, in the right doses, at the right intervals, for the right duration. 30000 DOTS providers have been recruited nationwide to provide treatment to the patients.

Under optimal programme conditions, treatment without observation achieves a success rate of $30-60 \%$, whereas, direct observation results in a much higher success rate of $85-95 \%$. DOTS helps to reduce development of drug resistance, because direct observation ensures adherence and hence reduces the probability of emergence of drug-resistant organisms. Further, following a correct treatment regimen reduces the spread of infection in the community and helps in controlling development of new cases.

To address the MDR-TB problem through appropriate management of patients and strategies to prevent the propagation and dissemination of MDR-TB, the Indian Government introduced DOTS-Plus programe under RNTCP in 2009 and issued DOTS-Plus guidelines promoting full integration of DOTS and DOTS-Plus activities so that patients with MDR-TB are both correctly identified and properly managed under this programme.

Under DOTS-plus programme RNTCP uses oral second line six anti TB drugs (Kanamycin, Ofloxacin, Ethionamide, Pyrazinamide, Ethambutol and Cycloserine) during 6-9 months of the Intensive Phase and 4 drugs (Ofloxacin, Ethionamide, Ethambutol and Cycloserine) during the 18 months of the Continuation Phase. $p$-aminosalicylic acid (PAS) is included in the regimen as a substitute drug if any bactericidal drug (Kanamycin, Ofloxacin, Z Pyrazinamide and Ethionamide) or 2 bacteriostatic (Ethambutol and Cycloserine) drugs are not tolerated. All the drugs used in DOTS and DOTS-plus strategies of RNTCP are manufactured in India.

\section{DIAGNOSTIC STANDARDS AND AVAILABILITY DIAGNOSTIC TESTS}

\section{HIV Diagnosis}

\section{Method}

All laboratories including those in the private sector undertaking HIV testing activities follow guidelines issued by the National AIDS Control Organization [12]. These guidelines provide all information regarding the diagnosis of HIV infection, monitoring of patients on ART etc.

\section{Observations}

India has well developed laboratory approaches for ensuring blood safety and donation safety (including tissue and organ); surveillance of high risk groups; sentinel surveillance; diagnosis and research. Most HIV antibody screening and supplemental testing is performed at various testing centres under NACO whereas Reference centres provide reference diagnostic services for problematic sera and perform assays like Western Blots, PCR and viral load assays etc.

Primary screening is done at Integrated Counselling and Testing (ICTC) by rapid tests. Rapid tests approved by the NACO for use as primary screening tests are based on the principle of EIA or particle agglutination. These are provided free of cost to all ICTCs and other testing centres under NACO. Samples found positive in primary screening are further tested by two different EIAs/ELISAs or by Western Blot assay for validation of results. This service is provided free of cost to all individuals reporting at ICTCs.

Since HIV infection in infants $(<18$ months) born to HIV-infected mothers cannot be established on the basis of serological tests because of transplacentally transmitted maternal antibodies HIV specific DNA PCR is recommended. Facility for such a test is provided at all the Reference centres by the NACO. These tests are also provided free of cost only in these centres.

Although NACO recommends CD4+ T-cell enumeration and HIV plasma viral load by real time PCR assay for monitoring patients on ART, it has been providing only CD4+ T-cell enumeration to all patients free of costs.

\section{Tuberculosis Diagnosis}

Sputum microscopy continues to be the primary tool for detection of infectious TB, as it provides information on the extent of infection of the patient, helps in categorization of the patient for treatment and is an objective method to monitor the patient's progress. Moreover, the result is available within two days and the correct treatment can be started immediately. Apart from sputum microscopy, RNTCP also uses standardized diagnostic algorithms to diagnose and treat all forms of TB wherein X-ray plays a supporting role (Fig. 6).

\section{MDR/XDR-TB Diagnosis}

A major limiting factor in making State wise representative data on MDR/XDR-TB is the lack of quality assured culture and drug susceptibility testing (DST) laboratory facilities. It is important to note that the diagnosis of MDR/XDR-TB is laboratory based. The programme is in the process of establishing a network of 27 accredited Culture and Drug Susceptibility testing Intermediate Reference Laboratories (IRLs) across the country in a phased manner for diagnosis and follow up of MDR-TB patients. Six culture and DST Labs including 6 IRLs (Andhra Pradesh, Delhi, Gujarat, Kerala, Maharashtra and Tamil $\mathrm{Nadu}$ ) and 2 private sector labs (BPRC Andhra Pradesh and CMC Vellore) have been accredited in 2008. Another 8 IRLs (Chattisgarh, Haryana, Jharkhand, Orissa, Rajasthan, Uttarakhand, Uttar Pradesh, West Bengal) and 5 medical college laboratories were under accreditation process and 


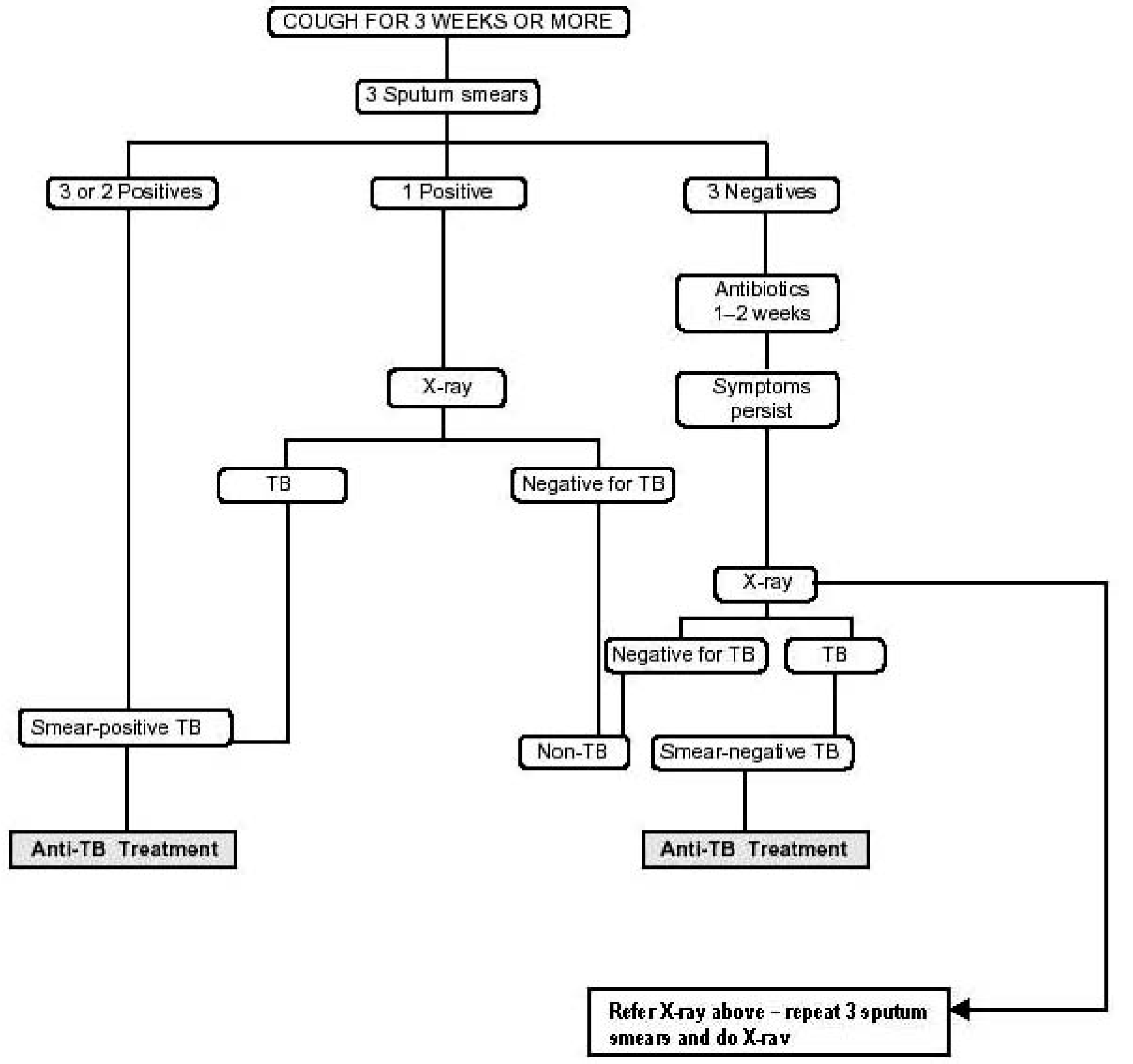

Fig. (6). Tuberculosis Diagnosis Algorithm (Adapted from RNTCP Status Report).

expected to be accredited in 2010. The remaining IRLs will be accredited in 2011.

\section{HIV/MTB CO-INFECTION}

The emergence and spread of HIV and drug-resistant tuberculosis further threaten to complicate the tuberculosis situation in the country. India, the third highest HIV burdened country, had an estimated 2.47 million people living with HIV/AIDS (PLHAs) in 2006 (that is, $0.36 \%$ of adult population in the country), emphasizing the enormous challenge ahead. All States and Union Territories of the country have reported HIV/AIDS patients. However, the HIV epidemic pattern shows great variance across the country. The worst affected states are Andhra Pradesh, Karnataka, Maharashtra, Manipur, Nagaland and Tamil Nadu. These six states have reported more than $75 \%$ of all the AIDS cases in India and are classified as High
Prevalence States. Three other states namely Goa, Gujarat and Pondicherry, have been classified as Moderate HIV prevalence states. Even within the high prevalence states, there are districts which have ante-natal HIV levels below $1 \%$.

Tuberculosis is one of the earliest opportunistic diseases to develop amongst persons infected with HIV. HIV infection is the most powerful risk factor for the progression of latent MTB infection to TB disease. An HIV positive person co-infected with MTB has $50-60 \%$ life time risk of developing TB disease, as compared to an HIV negative person who has a $10 \%$ life-time risk of developing TB disease. Thus TB mortality could well be influenced by the $\mathrm{MTB} / \mathrm{HIV}$ co-infection al least in certain districts in the country particularly in districts in India with high prevalence of HIV in TB patients. 
In India, the TB epidemic is pre-dominantly driven by the non-HIV positive TB cases. It is estimated that nearly $5 \%$ of all TB patients are infected with HIV. The periodic HIV survey in TB patients, which was carried out in 4 districts in 2005-06, was scaled up to 15 districts in 2006-07. The 2007 survey represents the most detailed evaluation to date of HIV epidemiology among TB patients in India. The survey demonstrated that the prevalence of HIV among TB patients varied substantially across the geographic regions between $1 \%$ and $13.8 \%$ across the 15 surveyed districts [13].

A national policy to coordinate common activities for HIV/AIDS and TB has been formulated by the National AIDS Control Organization and the Central TB Division [14]. TB and TB/HIV interventions are reciprocally included in the national policies of both programmes. TB/HIV coordination activities are conducted nationwide, and are being intensified in 9 states with districts considered to have the highest HIV burdens in the country. Training and sensitization of all health staff is being conducted using jointly developed TB/HIV training modules. Surveillance for HIV infection among TB patients that has been previously conducted in special surveys now relies upon routine reporting of HIV status of TB patients. In 20080.16 million HIV positive individuals were referred to RNTCP programme for MTB detection and 0.13 million MTB infected individuals were referred to ICTC (Integrated Counselling and treatment Centres) for HIV detection. 35000 and 20000 patients were found to be infected with MTB and HIV, respectively.

In response to the dual epidemics of AIDS and TB, the World Health Organization (WHO) has recommended has recommended guidelines for AIDS and TB prevention, care and treatment services. These include interventions that reduce the morbidity and mortality from TB in people living with HIV, such as the provision of antiretroviral therapy (ART) and the Three I's for HIV/TB: intensified casefinding of TB (ICF), isoniazid preventive therapy (IPT), and infection control for TB. These guidelines emphasize that IPT is a core component of HIV prevention and care, and should be the primary responsibility of AIDS programmes and HIV service providers. In addition, the provision of IPT should not be viewed as an isolated intervention for people living with HIV. Rather, it should be part of a TB prevention package along with infection control for TB, ICF and provision of ART. However, in contrast with many other countries, isoniazid preventative therapy is not recommended in clinical care guidelines for human immunodeficiency virus (HIV)-infected persons with latent tuberculosis (TB) in India.

\section{ACKNOWLEDGEMENTS}

The work presented in this manuscript is derived from the EUCO-Net project funded by the $7^{\text {th }}$ Framework
Programme of the European Commission. Author of this report was Core group expert on HIV and Tuberculosis from India in this project. He was very ably assisted by additional experts (Dr. Naveet Wig, Dr. Kamini Walia and Dr. Akash Gulalia) in collection of information used for preparation of the Country report and this manuscript. Collation of information and assistance in writing the manuscript by Kartik Yadav is greatly appreciated.

\section{REFERENCES}

[1] Census of India, Registrar General \& Census Commissio-ner, India, Ministry of Home Affairs, Government of India 2001 (available at http://censusindia.gov.in)

[2] HIV Sentinel Surveillance and HIV Estimation in India 2007: A technical brief. National AIDS Control Organization, Ministry of Health and Family Welfare, Government of India 2008 (available at http://www.nacoonline.org)

[3] Annual Report 2008-2009. National AIDS Control Organization Ministry of Health and Family Welfare, Government of India 2010 (available at http://www.nacoonline.org)

[4] TB India 2009: RNTCP Status Report Central TB Division, Directorate General of Health Services, Ministry of Health and Family Welfare, Nirman Bhawan, New Delhi-110001. 2009 (available at http://www.tbcindia.org)

[5] TB India 2010: RNTCP Status Report Central TB Division, Directorate General of Health Services, Ministry of Health and Family Welfare, Nirman Bhawan, New Delhi-110001, 2010 (available at http://www.tbcindia.org)

[6] Technical and Operational Guidelines for Tuberculosis Control. Central TB Division, Directorate General of Health Services, Ministry of Health and Family Welfare, Nirman Bhawan, New Delhi-110001, 2005 (available at http://www.tbcindia.org)

[7] Guidelines for Quality Assurance of smear microscopy for diagnosing tuberculosis.. Central TB Division, Directorate General of Health Services, Ministry of Health and Family Welfare, Nirman Bhawan, New Delhi-110001. 2005 (available at http:// www.tbcindia.org)

[8] DOTS-Plus Guidelines. Central TB Division, Directorate General of Health Services, Ministry of Health and Family Welfare, Nirman Bhawan, New Delhi-110001. 2010 (available at http:// www.tbcindia.org)

[9] V.G. Rao, P.G. Gopi, J. Bhat, R. Yadav, D.F. Wares. Role of BCG vaccination in tuberculosis control. Curr Sci 2009; 96: 1307-8.

[10] Antiretroviral Therapy Guidelines for HIV-infected Adults and Adolescents Including Post-exposure Prophylaxis. National AIDS Control Organization, Ministry of Health and Family Welfare, Government of India 2007 (available at http://www.naco online.org)

[11] National Guidelines on Second-line ART for adults and adolescents. National AIDS Control Organization, Ministry of Health and Family Welfare, Government of India 2008 (available at http://www. nacoonline.org)

[12] Guidelines on HIV Testing. National AIDS Control Organization, Ministry of Health and Family Welfare, Government of India 2007 (available at http://www.nacoonline.org)

[13] The HIV-TB Co-infection.. National AIDS Control Organization \& Central TB Division. Ministry of health and Family Welfare, Government of India 2008 (available at http://www.naco online.org)

[14] National Framework for Joint TB/HIV Collaborative Activities. Central TB Division and National AIDS Control Organization, Ministry of Health and Family Welfare, Government of India 2008 (available at http://www.tbcindia.org) 